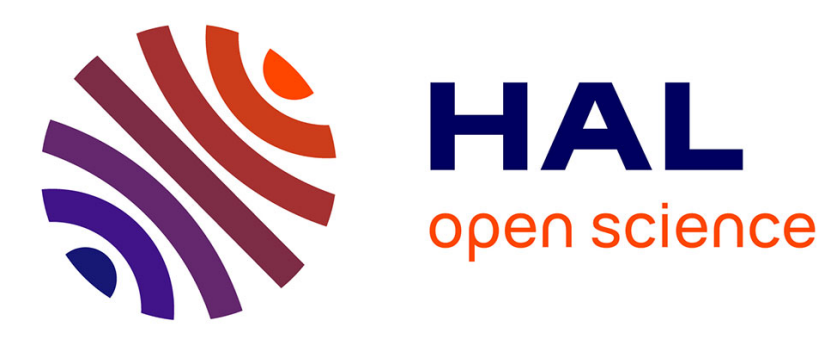

\title{
Superconfined falling liquid films: linear versus nonlinear dynamics
}

Gianluca Lavalle, Sophie Mergui, Nicolas Grenier, Georg F. Dietze

\section{To cite this version:}

Gianluca Lavalle, Sophie Mergui, Nicolas Grenier, Georg F. Dietze. Superconfined falling liquid films: linear versus nonlinear dynamics. Journal of Fluid Mechanics, 2021, 919 (R2), 10.1017/jfm.2021.417 . hal-03236774

\section{HAL Id: hal-03236774 https://hal.science/hal-03236774}

Submitted on 18 Aug 2021

HAL is a multi-disciplinary open access archive for the deposit and dissemination of scientific research documents, whether they are published or not. The documents may come from teaching and research institutions in France or abroad, or from public or private research centers.
L'archive ouverte pluridisciplinaire HAL, est destinée au dépôt et à la diffusion de documents scientifiques de niveau recherche, publiés ou non, émanant des établissements d'enseignement et de recherche français ou étrangers, des laboratoires publics ou privés. 


\section{Superconfined falling liquid films: linear versus nonlinear dynamics}

\begin{tabular}{|c|c|}
\hline Journal: & Journal of Fluid Mechanics \\
\hline Manuscript ID & JFM-21-RP-0089.R2 \\
\hline mss type: & JFM Rapids \\
\hline $\begin{array}{r}\text { Date Submitted by the } \\
\text { Author: }\end{array}$ & 30-Apr-2021 \\
\hline Complete List of Authors: & $\begin{array}{l}\text { Lavalle, Gianluca; UMR5307 } \\
\text { Grenier, Nicolas; Laboratoire d'Informatique pour la Mecanique et les } \\
\text { Sciences de I'Ingenieur } \\
\text { Mergui, Sophie; Fluides Automatique et Systemes Thermiques } \\
\text { Dietze, Georg; CNRS, Laboratoire FAST - UMR } 7608\end{array}$ \\
\hline JFM Keywords: & $\begin{array}{l}\text { Thin films < Interfacial Flows (free surface), Instability, Gas/liquid flows } \\
<\text { Multiphase and Particle-laden Flows }\end{array}$ \\
\hline Abstract: & $\begin{array}{l}\text { The effect of a counter-current gas flow on the linear stability of an } \\
\text { inclined falling liquid film switches from destabilizing to stabilizing, as the } \\
\text { flow confinement is increased. We confront this linear effect with the } \\
\text { response of nonlinear surface waves resulting from long-wave interfacial } \\
\text { instability. For the strongest confinement studied, the gas flow damps } \\
\text { both the linear growth rate and the amplitude of nonlinear travelling } \\
\text { waves, and this holds for waves of the most-amplified frequency and for } \\
\text { low-frequency solitary waves. In the latter case, waves are shaped into } \\
\text { elongated humps with a flat top that resist secondary instabilities. For } \\
\text { intermediate confinement, the linear and nonlinear responses are } \\
\text { opposed and can be non-monotonic. The linear growth rate of the most- } \\
\text { amplified waves first decreases and then increases as the gas velocity is } \\
\text { increased, whereas their nonlinear amplitude is first amplified and then } \\
\text { damped. Conversely, solitary waves are amplified linearly but damped } \\
\text { nonlinearly. For the weakest confinement, solitary waves are prone to } \\
\text { two secondary instability modes that are not observed in unconfined } \\
\text { falling films. The first involves waves of diminishing amplitude } \\
\text { slipstreaming toward their growing leading neighbours. The second } \\
\text { causes wave splitting events that lead to a train of smaller, shorter } \\
\text { waves. }\end{array}$ \\
\hline
\end{tabular}

\section{SCHOLARONE Manuscripts}


Banner appropriate to article type will appear here in typeset article

1 Superconfined falling liquid films: linear versus 2 nonlinear dynamics

3 Gianluca Lavalle ${ }^{1}$, Sophie Mergui ${ }^{2,4}$, Nicolas Grenier ${ }^{3}$, and Georg F. Dietze ${ }^{2} \dagger$

${ }^{1}$ Mines Saint-Etienne, Univ Lyon, CNRS, UMR 5307 LGF, Centre SPIN, F-42023 Saint-Etienne, France.

${ }^{2}$ Université Paris-Saclay, CNRS, FAST, 91405, Orsay, France.

${ }^{3}$ Université Paris-Saclay, CNRS, LISN, 91405, Orsay, France.

${ }^{4}$ Sorbonne Université, Faculté des Sciences et Ingénierie, UFR d’Ingénierie, F-75005 Paris, France.

(Received xx; revised xx; accepted xx)

9 The effect of a counter-current gas flow on the linear stability of an inclined falling liquid film switches from destabilizing to stabilizing, as the flow confinement is increased. We confront this linear effect with the response of nonlinear surface waves resulting from long-wave interfacial instability. For the strongest confinement studied, the gas flow damps both the linear growth rate and the amplitude of nonlinear travelling waves, and this holds for waves of the most-amplified frequency and for low-frequency solitary waves. In the latter case, waves are shaped into elongated humps with a flat top that resist secondary instabilities. For intermediate confinement, the linear and nonlinear responses are opposed and can be non-monotonic. The linear growth rate of the most-amplified waves first decreases and then increases as the gas velocity is increased, whereas their nonlinear amplitude is first amplified and then damped. Conversely, solitary waves are amplified linearly but damped nonlinearly. For the weakest confinement, solitary waves are prone to two secondary instability modes that are not observed in unconfined falling films. The first involves waves of diminishing amplitude slipstreaming toward their growing leading neighbours. The second causes wave splitting events that lead to a train of smaller, shorter waves.

Key words: Thin films

\section{Introduction}

We consider a gravity-driven two-dimensional liquid film falling down a plane tilted at an angle $\phi$ w.r.t. the horizontal, in contact with a counter-current gas flow that is strongly confined by an upper wall placed at $y=H$ (figure 1). Both fluids are Newtonian with constant fluid properties and the flow is laminar. Confined falling liquid films occur in rectification columns for cryogenic air separation, which contain structured packings that subdivide the column cross-section into millimetric channels (Valluri et al. 2005). Even stronger confinement is realized in compact reflux condensers (Vlachos et al. 2001), falling

$\dagger$ Email address for correspondence: dietze@ fast.u-psud.fr 


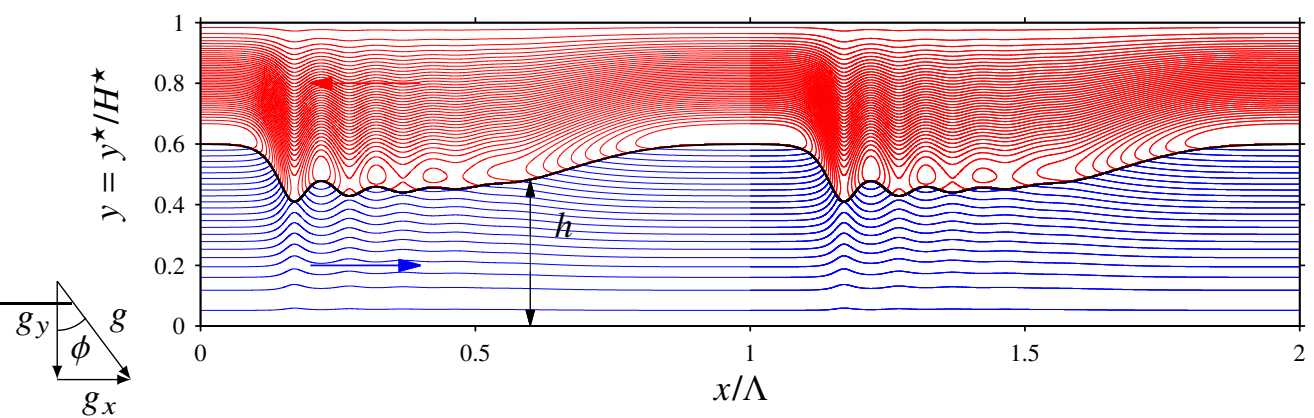

Figure 1: Problem sketch: gravity-driven falling liquid film (lower blue streamlines) in contact with a counter-current gas flow (upper red streamlines) flowing through a channel of dimensional gap height $H^{\star}$ inclined at an angle $\phi$ w.r.t. the horizontal. Streamlines (separated by constant stream function increments) are shown in the wall-fixed reference frame, and $\Lambda$ is the wavelength.

film microreactors (Zhang et al. 2009), and micro-gap coolers (Kabov et al. 2011). We are interested in nonlinear waves that form on the surface of the falling liquid film due to the long-wave Kapitza instability (Kapitza 1948), and, in particular, how these are affected by the gas velocity in a strongly-confined setting. Such waves are known to trigger flooding events, either by local obstruction of the channel, flow reversal, or wave reversal (Vlachos et al. 2001; Trifonov 2010a; Tseluiko \& Kalliadasis 2011).

For weak confinements, flooding seems to be favoured by decreasing the gap height and/or increasing the gas flow rate. Experiments (Kofman et al. 2017) and numerical simulations (Trifonov 2010a,b) alike have shown that the amplitude of nonlinear waves increases with increasing counter-current gas flow and that this growth diverges in the vicinity of the flooding point (Drosos et al. 2006). Moreover, actual flooding experiments have shown that the critical gas flow rate decreases with diminishing gap height (Sudo 1996). Linear stability investigations, which demonstrate an increase in the maximal linear growth rate with increasing gas velocity, tend to confirm this nonlinear picture (Alekseenko et al. 2009; Vellingiri et al. 2015; Schmidt et al. 2016; Trifonov 2017).

On the other hand, recent investigations suggest that strong confinements may, in fact, lower the risk of flooding. Lavalle et al. (2019) have shown that the Kapitza instability can be entirely suppressed by sufficiently confining the gas, as suggested by Tilley et al. (1994) and confirmed by Kushnir et al. (2021), and that this is facilitated by low tilt angles. Further, the authors observed that the linear stabilization, which they confirmed experimentally, is amplified by increasing the counter-current gas flow rate. Recent nonlinear direct numerical simulations (DNS) of inclined falling liquid films (Trifonov 2020) have identified a nonmonotonic variation of the interfacial velocity, mean film thickness, and inter-phase friction coefficient with increasing counter-current gas velocity, although the trend of the wave amplitude remained monotonic and increasing.

These investigations have motivated us to take a closer look at strongly-confined inclined falling liquid films, in contrast to Dietze \& Ruyer-Quil (2013) and Lavalle et al. (2020), who studied the vertical configuration, where the gas-induced linear stabilization is relatively weak. This is because the inertia-induced destabilizing mechanism of the Kapitza instability is weakened less and less by the stabilizing effect of normal gravity as the tilt angle is increased, and thus the relative weight of the gas effect diminishes (Lavalle et al. 2019). We aim to confront linear stability predictions with the response of nonlinear surface waves to an increasingly strong counter-current gas flow. In particular, we wish to know whether nonlinear travelling waves can be damped under the effect of the gas flow, in line with the 
linear observations, and, if so, whether they may resist secondary instability. Such a situation would amount to a reduced flooding risk. By secondary instability we mean the loss of stability of travelling-wave solutions (TWS) produced by the primary Kapitza instability (Liu \& Gollub 1993; Lavalle et al. 2020), and our analysis is restricted to two-dimensional such instability modes.

An enticing preliminary result was obtained by Samanta (2014), who showed that applying a constant interfacial shear stress to an inclined wavy falling liquid film can strongly reduce the amplitude of nonlinear surface waves. However, for the strong confinement studied here, variations of the shear stress with wave height play an important role (Lavalle et al. 2019), and the gas pressure gradient, which was also neglected in the model of Samanta (2014), needs to be accounted for (Dietze \& Ruyer-Quil 2013).

To tackle this problem, we use the two-phase weighted residual integral boundary layer (WRIBL) model of Dietze \& Ruyer-Quil (2013) to construct TWS, with the continuation software Auto07P (Doedel 2008), and to compute spatially evolving wavy falling liquid films, with our own finite-difference code (Lavalle et al. 2020). These nonlinear computations are confronted with linear stability calculations based on the WRIBL model, and solutions of the full Orr-Sommerfeld (OS) eigenvalue problem (Tilley et al. 1994), whereby we have employed a spatial stability formulation (Barmak et al. 2016). Also, we check for periodic secondary instabilities via transient periodic computations started from TWS (Lavalle et al. 2020), and confront our model with a direct numerical simulation (DNS) based on the full Navier-Stokes equations, using the finite-volume solver Basilisk (Popinet 2015).

Our manuscript is structured as follows. The mathematical description and numerical methods are introduced in $\$ 2$, followed by section 3, which reports results of our linear and nonlinear computations. Subsection 3.1 is dedicated to surface waves of the linearly mostamplified frequency, whereas subsection 3.2 concerns low-frequency solitary waves (here, we will also introduce our DNS data). Conclusions are drawn in section 4.

\section{Mathematical description}

The flow in figure 1 is governed by the Navier-Stokes and continuity equations, written in Einstein notation using the directional indices $i=1,2$ and $j=1,2\left(x_{1}=x, u_{1}=u, x_{2}=y\right.$, and $\left.u_{2}=v\right)$, and the phase indicator $m$, which identifies liquid $(m=l)$ and gas $(m=g)$ :

$$
\begin{gathered}
X_{m} \partial_{t} u_{i}+u_{j} \partial_{x_{j}} u_{i}=-\partial_{x_{i}} p_{m}+\operatorname{Re}_{m}^{-1} \partial_{x_{j} x_{j}} u_{i}+X_{m}^{2} \mathrm{Fr}^{-2}\left\{\delta_{i 1} \sin (\phi)-\delta_{i 2} \cos (\phi)\right\}, \\
\partial_{x_{j}} u_{j}=0,
\end{gathered}
$$

where lengths have been scaled with the channel height $\mathcal{L}=H^{\star}$ (stars denote dimensional quantities throughout), velocities with the phase-specific signed superficial velocities $\mathcal{U}_{m}=q_{m 0}^{\star} / H^{\star}$, time with $\mathcal{T}=\mathcal{L} / \mathcal{U}_{l}$, and the phase-specific pressure $p_{m}$ with $\rho_{m} \mathcal{U}_{m}^{2}$. Further, $\delta_{i j}$ is the Kronecker symbol, $X_{l}=1$, and $X_{g}=\mathcal{U}_{l} / \mathcal{U}_{g}$. The gravitational acceleration $g$ enters through the Froude number $\mathrm{Fr}=\mathcal{U}_{l} / \sqrt{g \mathcal{L}}$, and the Reynolds numbers $\operatorname{Re}_{m}=\mathcal{U}_{m} \mathcal{L} \frac{\rho_{m}}{\mu_{m}}=q_{m 0}^{\star} \frac{\rho_{m}}{\mu_{m}}$ are based on the phase-specific signed nominal flow rates $q_{m 0}^{\star}$ of the flat-film primary flow, $q_{g 0}^{\star}$ and $\operatorname{Re}_{g}$ being negative for a counter-current gas flow. The boundary conditions are:

$$
\left.u_{l}\right|_{y=0}=\left.v_{l}\right|_{y=0}=\left.u_{g}\right|_{y=1}=\left.v_{g}\right|_{y=1}=0,
$$

and the kinematic and dynamic coupling conditions at the film surface $y=h(x, t)$ :

$$
u_{l}=X_{g}^{-1} u_{g}, \quad v_{l}=X_{g}^{-1} v_{g}=\partial_{t} h+u_{l} \partial_{x} h,
$$


109

$$
\begin{gathered}
p_{l}+\left[S_{i j}^{l} n_{j}\right] n_{i}=X_{g}^{-2} \Pi_{\rho} p_{g}+X_{g}^{-1} \Pi_{\mu}\left[S_{i j}^{g} n_{j}\right] n_{i}+\text { We } \kappa, \\
{\left[S_{i j}^{l} n_{j}\right] \tau_{i}=X_{g}^{-1} \Pi_{\mu}\left[S_{i j}^{g} n_{j}\right] \tau_{i},}
\end{gathered}
$$

where $S_{i j}^{m}=\frac{1}{2}\left(\partial_{x_{j}} u_{i}+\partial_{x_{i}} u_{j}\right)$ denotes the strain rate tensor, $\Pi_{\mu}=\mu_{g} / \mu_{l}$ and $\Pi_{\rho}=\rho_{g} / \rho_{l}$ are the dynamic viscosity and density ratios, and the surface tension $\sigma$ enters through the Weber number We $=\sigma \rho_{l}^{-1} \mathcal{U}_{l}^{-2} \mathcal{L}^{-1}$. The orthonormal surface coordinate system is constructed by $\boldsymbol{n}=\left[-\partial_{x} h, 1\right]\left(1+\partial_{x}^{2} h\right)^{-1 / 2}$ and $\tau=\left[1, \partial_{x} h\right]\left(1+\partial_{x}^{2} h\right)^{-1 / 2}$, from which we obtain the film surface curvature $\kappa=-\boldsymbol{\nabla} \cdot \boldsymbol{n}$.

We perform two types of calculations based on the first principles (2.1) to validate our low-dimensional model. First, we solve the OS linear stability problem (Tilley et al. 1994), assuming spatially growing normal modes (Barmak et al. 2016):

$$
\left[\begin{array}{c}
h \\
\Phi \\
\Psi \\
p_{m}
\end{array}\right]=\left[\begin{array}{c}
h_{0} \\
\Phi_{0}(y) \\
\Psi_{0}(y) \\
p_{m 0}(x, y)
\end{array}\right]+\left[\begin{array}{c}
\hat{h} \\
\phi(y) \\
\psi(y) \\
\hat{p}_{m}(y)
\end{array}\right] \exp \{i(k x-\omega t)\},
$$

where $\Phi$ and $\Psi$ designate the stream functions in the liquid and gas, the subscript 0 denotes the flat-interface base flow, $k \in \mathbb{C}$ is the complex wave number of the perturbation, and $\omega \in \mathbb{R}$ its angular frequency. We focus on long-wave instability modes, which we track through numerical continuation using Auto07P (Lavalle et al. 2019), having checked with a Chebyshev collocation code (Barmak et al. 2016) that short wave modes remain stable throughout the studied parameter range. Second, we perform a DNS with the finite-volume solver Basilisk (Popinet 2015), based on the volume of fluid (VOF) and the continuum surface force (CSF) methods, following Dietze (2019). Here, we impose periodic conditions on a domain spanning the wavelength $\Lambda$.

Our low-dimensional model is based on the weighted residual integral boundary layer (WRIBL) approach (Ruyer-Quil \& Manneville 1998; Kalliadasis et al. 2012), which describes the flow via evolution equations for the flow rate $q$ and film height $h$. We employ the two-phase formulation of Dietze \& Ruyer-Quil (2013) written in Einstein notation ( $m=l, g$ and $n=l, g$ ):

$$
\left\{S_{m} \partial_{t} q_{m}+F_{m n} q_{m} \partial_{x} q_{n}+G_{m n} q_{j} q_{m} \partial_{x} h\right\}=- \text { We } \partial_{x x x} h
$$

$$
\begin{aligned}
& +\operatorname{Fr}^{-2}\left(1-\Pi_{\rho}\right)\left\{\sin (\phi)-\cos (\phi) \partial_{x} h\right\}+\operatorname{Re}_{m}^{-1} C_{m} q_{m} \\
& +\operatorname{Re}_{m}^{-1}\left\{J_{m} q_{m}\left(\partial_{x} h\right)^{2}+K_{m} \partial_{x} q_{m} \partial_{x} h+L_{m} q_{m} \partial_{x x} h+M_{m} \partial_{x x} q_{m}\right\},
\end{aligned}
$$

$$
\partial_{x} q_{l}+\partial_{t} h=0, \quad \partial_{x} q_{g}-X_{g} \partial_{t} h=0,
$$

where $q_{l}$ and $q_{g}$ denote the liquid and gas flow rates (per unit width) and the coefficients $S_{m}, F_{m n}, G_{m n}, C_{m n}, J_{n}, K_{n}, L_{n}$, and $M_{n}$ are known functions of the film height $h$ (Dietze \& Ruyer-Quil 2013).

We perform linear stability calculations by solving the dispersion equation $\operatorname{DR}(\omega, k)=0$, obtained by linearizing (2.3) around $\left[h_{0}, q_{l 0}, q_{g 0}\right]$, for $k=k_{r}+i k_{i}$ at a given $\omega \in \mathbb{R}$ :

$$
\begin{aligned}
& {\left[h, q_{l}, q_{g}\right]^{T}=\left[h_{0}, q_{0 l}, q_{0 g}\right]^{T}+\left[\hat{h}, \hat{q}_{l}, \hat{q}_{g}\right]^{T} \exp \{i(k x-\omega t)\},} \\
& \text { Cambridge University Press }
\end{aligned}
$$


145

$$
\begin{aligned}
\mathrm{DR} & =i \omega^{2}\left\{S_{g}-S_{l}\right\}+i k \omega\left\{F_{m l} q_{m}-F_{m g} q_{m}\right\}+i k^{2} G_{m n} q_{m} q_{n} \\
& +i k^{2} \mathrm{Fr}^{-2}\left\{\cos (\phi)-\Pi_{\rho} \cos (\phi)\right\}-i^{3} k^{4} \mathrm{We}+\omega\left\{\operatorname{Re}_{g}^{-1} C_{g}-\mathrm{Re}_{l}^{-1} C_{l}\right\} \\
& -k \operatorname{Re}_{m}^{-1} \partial_{h} C_{m} q_{m}-i^{2} k^{3} \operatorname{Re}_{m}^{-1} L_{m} q_{m}+i^{2} k^{2} \omega\left\{\operatorname{Re}_{g}^{-1} M_{g}-\operatorname{Re}_{l}^{-1} M_{l}\right\} .
\end{aligned}
$$

We also compute nonlinear travelling-wave solutions (TWS), which remain unaltered in a reference frame moving at the wave speed $c$, through numerical continuation based on (2.3), using Auto07P (Doedel 2008). Our code allows to track TWS at the linearly most-amplified angular frequency $\omega=\omega_{\max }$, via the following constraints (Dietze et al. 2020):

$$
\operatorname{DR}\left(\omega_{\max }, k\right)=0,\left.\quad \partial_{\omega} k_{i}\right|_{\omega=\omega_{\max }}=0 .
$$

Finally, we check the stability of nonlinear TWS via transient computations based on (2.3), using either periodic or inlet/outlet boundary conditions (Lavalle et al. 2020).

\section{Results}

We set the tilt angle to $\phi=10^{\circ}$ and focus on a single fluid combination, a $83 \%$ by weight aqueous dimethylsulfoxide (DMSO) solution used in experiments (Dietze et al. 2009), where $\rho_{l}=1098.3 \mathrm{~kg} / \mathrm{m}^{3}, \mu_{l}=3.13 \mathrm{mPas}$, and $\sigma=0.0484 \mathrm{~N} / \mathrm{m}$, in contact with ambient air. The Kapitza number for this combination is $\mathrm{Ka}=\sigma \rho_{l}^{-1 / 3} g^{-1 / 3} \mu_{l}^{-4 / 3}=509.5$. The channel height $H^{\star}$ is varied as $H^{\star}=1.2,1.7,1.8,1.9,2.1$, and $2.4 \mathrm{~mm}$, which corresponds to values of $\eta=2$, $2.8,3,3.1,3.4$, and 3.9 for the relative confinement:

$$
\eta=H^{\star} /\left.h_{0}^{\star}\right|_{M=1}=1 /\left.h_{0}\right|_{M=1},
$$

where $\left.h_{0}\right|_{M=1}$ is the primary flow film thickness for an aerostatic gas pressure gradient, i.e. $M=\partial_{x} p_{g} / \sin (\phi)=1$. We wish to confront the linear and nonlinear implications of increasing the counter-current gas flow rate at fixed $\mathrm{Re}_{l}$. In particular, we wish to know whether nonlinear waves can be damped via increasing $\left|\operatorname{Re}_{g}\right|$.

\subsection{Most-amplified waves}

Figure 2 demonstrates the effect of increasing the counter-current gas flow rate on the linearly most-amplified waves $\left(\omega=\omega_{\max }\right)$ at fixed $\operatorname{Re}_{l}=15$ for different $\eta$ values. Along each curve in panels $2 \mathrm{a}$ and $2 \mathrm{c}$, the channel height $H^{\star}$ remains fixed while $h_{0}$ increases (between $10 \%$ for the strongest and $20 \%$ for the weakest confinement), and so $\eta$ (3.1) specifies a representative confinement for each case, corresponding to the rightmost point of each curve (where $M=1$ ). Curves in panel 2a track the maximum linear spatial growth rate $-k_{i}^{\max }$ in terms of $\mathrm{Re}_{g}$, dashed lines corresponding to OS and solid lines to WRIBL calculations. At the largest $\eta$ (filled squares, $\eta=3.9$ ), the growth rate increases monotonically with $\left|\operatorname{Re}_{g}\right|$, implying a gas-induced destabilization, up to the onset of absolute instability (AI), where $-k_{i \max }$ diverges (Vellingiri et al. 2015). Conversely, at very small $\eta$ values (open squares and pentagons, $\eta=2,2.8$ ), the effect of the gas is monotonically stabilizing, up to the point of fully suppressing (S) the long-wave Kapitza instability (Lavalle et al. 2019; Kushnir et al. 2021). In the intermediate range (crosses, asterisks, and diamonds, $\eta=3,3.1$, and 3.4), the behaviour is non-monotonic, stabilization occurring at low and destabilization at large values of $\left|\operatorname{Re}_{g}\right|$. Panels $2 \mathrm{~b}(\eta=3.1)$ and $2 \mathrm{~d}(\eta=2)$ represent dispersion curves for the non-monotonic and fully-stabilizing cases. Overall, there is quantitative agreement for $\left|\operatorname{Re}_{g}\right|<150$ between linear OS and WRIBL predictions in panels $2 \mathrm{a}, 2 \mathrm{~b}$, and $2 \mathrm{~d}$, whereas qualitative agreement is retained when approaching the AI limits. 


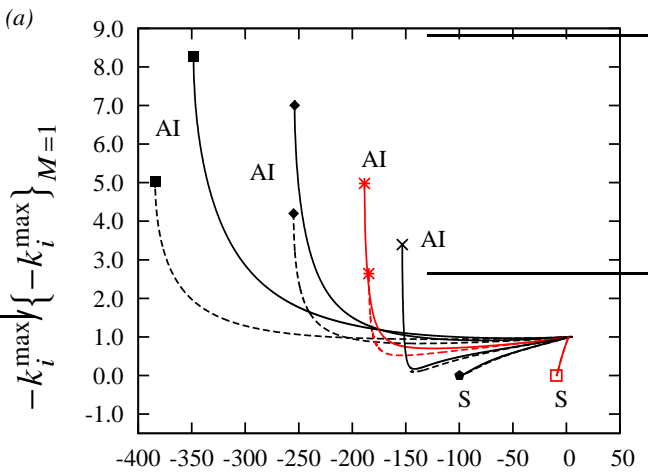

$\operatorname{Re}_{g}$

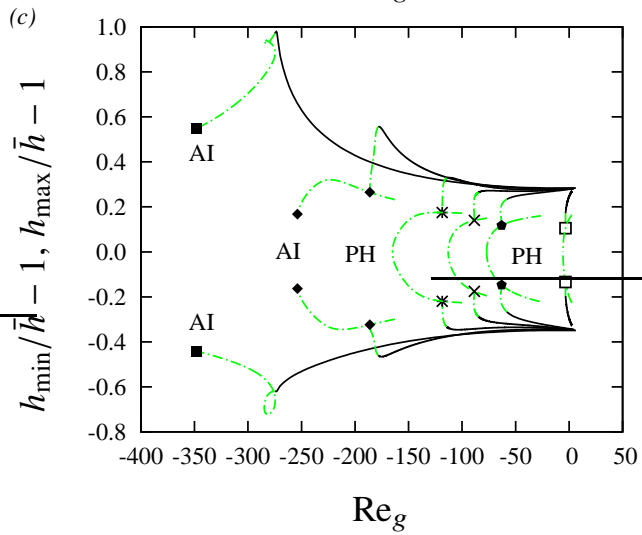

(b)

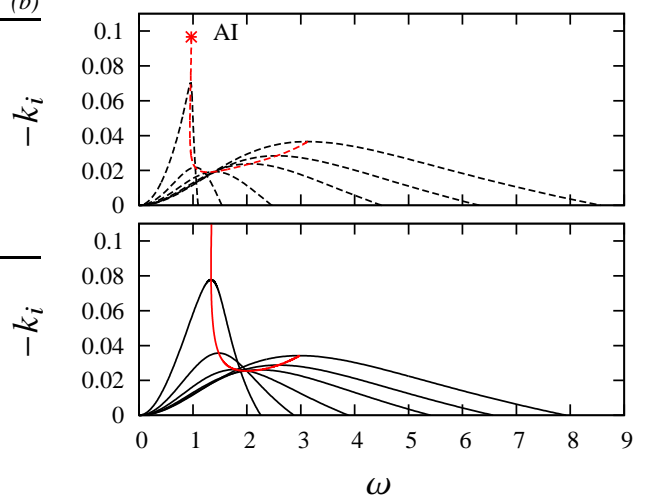

(d)

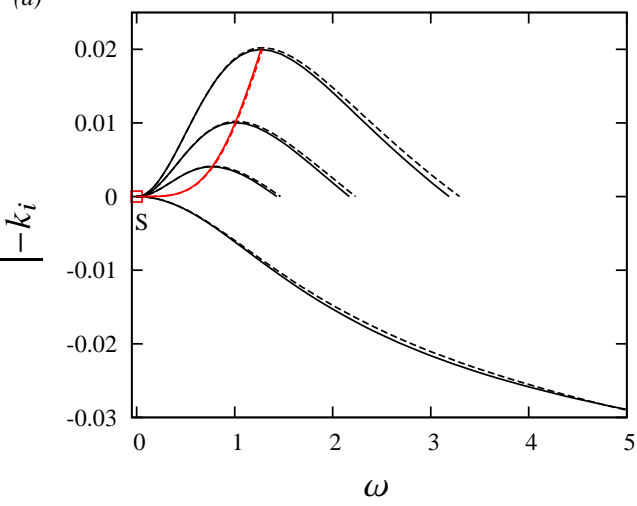

Figure 2: Most-amplified waves: $\phi=10^{\circ}, \operatorname{Re}_{l}=15, \mathrm{Ka}=509.5$. Linear (panels $a, b, d$ ) versus nonlinear (panel $c$ ) predictions. Filled squares: $\eta=3.9$, diamonds: $\eta=3.4$, asterisks: $\eta=3.1$, crosses: $\eta=3$; pentagons: $\eta=2.8$; open squares: $\eta=2$. (a) Maximal linear growth rate $-k_{i}^{\max }$ versus $\operatorname{Re}_{g}$, related to the aerostatic limit $\left\{-k_{i}^{\max }\right\}_{M=1}$, where $M=\partial_{x} p_{g} / \sin (\phi)$. Solid: WRIBL, dashed: OS; $(b, d)$ dispersion curves $-k_{i}(\omega)$ for two cases from panel $a$. Red curves trace $-k_{i}^{\max }\left(\omega_{\max }\right)$ up to absolute instability (AI) or full stabilization (S). (b) $\eta=3.1$; from right to left: $M=1, \operatorname{Re}_{g}=-60,-100,-145,-170$, and $-184 ;(d) \eta=2$; from right to left: $M=1, \operatorname{Re}_{g}=-4,-7$, and -10 ; (c) amplitude of nonlinear TWS (WRIBL) at $\omega=\omega_{\max }$. PH denotes period-halving bifurcations and dot-dashed green lines identify periodically unstable TWS.

Panel 2c plots the upper and lower relative film height deflections $h_{\max } / \bar{h}-1$ and $h_{\min } / \bar{h}-1$ for nonlinear TWS at $\omega=\omega_{\max }$, where $\bar{h}=\Lambda^{-1} \int_{0}^{\Lambda} h d x$ is the film height averaged over one wavelength, with $\bar{h} \neq h_{0}$ in the case of nonlinear waves. For $\eta=3,3.1$, and 3.4, TWS display a non-monotonic trend that is opposed to the linear one. That is, $h_{\max } / \bar{h}-1$ in panel 2c, which we will refer to as the wave amplitude, first increases and then decreases with increasing $\left|\operatorname{Re}_{g}\right|$, whereas $-k_{i}^{\max } /\left\{-k_{i}^{\max }\right\}_{M=1}$ in panel 2a first decreases and then increases. Conversely, for $\eta=2$ and 2.8, the nonlinear and linear trends both imply stabilization, and, for $\eta=3.9$, they both imply destabilization, at least up to the amplitude maximum in panel 2c. Except for the two weakest confinements $(\eta=2,2.8,3,3.1)$, TWS are bounded by a nonlinear wave suppression, where $h_{\max }=h_{\min }$, resulting from period-halving $(\mathrm{PH})$ bifurcations (marked by symbols), which sets in before the linear AI and S thresholds in panel 2a. Panel 3a shows wave profiles leading up to such a PH bifurcation $(\eta=3)$. The sole precursory capillary ripple is seen to grow until splitting the wave into two identical halves. Conversely, for $\eta=3.9$ (panel $3 b)$, the capillary ripple disappears when increasing $\left|\operatorname{Re}_{g}\right|$ toward the AI limit.

We conclude from figure 2 that linear stability predictions can be misleading. In particular, 

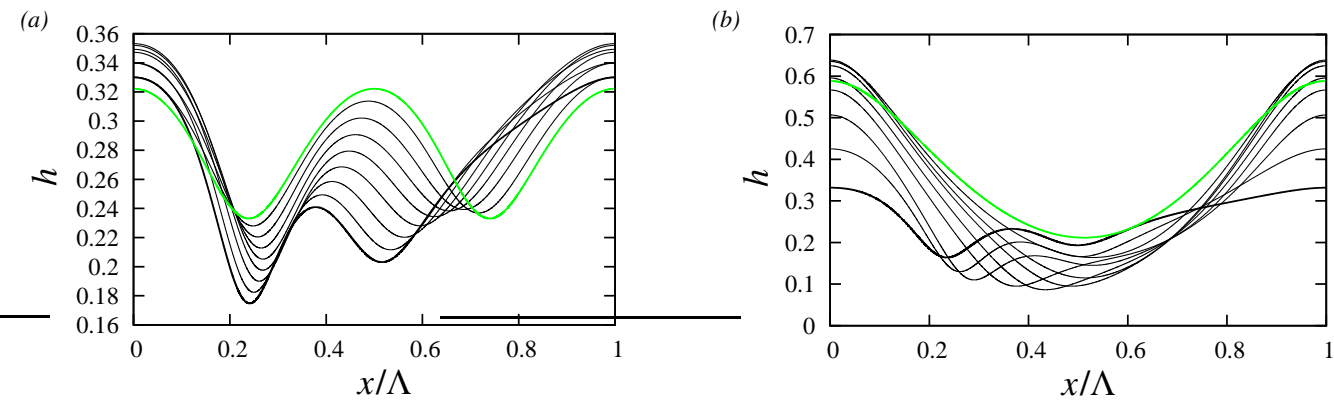

Figure 3: Wave profiles of TWS from panel 2c. (a) $\eta=3$ (cross in panel 2c). Approaching the PH bifurcation: $\operatorname{Re}_{g}=-37$ (thick solid) to $\operatorname{Re}_{g}=-88$ (green); (b) $\eta=3.9$ (filled square in panel 2c). Suppression of the capillary ripple while approaching the AI limit: $\operatorname{Re}_{g}=-79$ (thick solid) to $\mathrm{Re}_{g}=-348$ (green).

the amplitude of nonlinear waves may grow with increasing counter-current gas velocity, even though the linear growth rate decreases. Further, TWS become unstable to periodic secondary instability modes (dot-dashed lines in panel $2 \mathrm{c}$ ) beyond a threshold $\mathrm{Re}_{g}$, which we have determined via transient periodic computations started from TWS. These periodic modes do not lead to dangerous events, but TWS are also prone to a subharmonic instability in the case of a spatially-evolving film (Movie1.avi). Originally identified in unconfined films (Liu \& Gollub 1993), this instability triggers wave coalescence events (Chang et al. 1996a) that can lead to intermittent flooding in long channels (Dietze \& Ruyer-Quil 2013).

\subsection{Solitary waves}

We focus now on low-frequency solitary waves at a fixed wavelength $\Lambda=4.5 \tilde{\Lambda}_{\max }$, where $\tilde{\Lambda}_{\max }$ denotes the linearly most-amplified wavelength for a passive outer phase, all other parameters remaining as in figure 2 . These waves lie on the ascending branch of the linear dispersion curves in panels $2 \mathrm{~b}$ and $2 \mathrm{~d}$, and thus the linear effect of increasing the gas flow is monotonous, either destabilizing $(\eta=3,3.1,3.4,3.9)$ or stabilizing $(\eta=2,2.8)$. Panel $4 \mathrm{a}$ represents the nonlinear response of solitary TWS, evidencing a monotonous gas-induced attenuation of the wave amplitude for $\eta=2,2.8,3$, and 3.1. For $\eta=3$ and 3.1, this nonlinear effect is opposed to the linear amplification, and both effects are inverted w.r.t. the initial response of the most-amplified waves (panels 2a, 2c). Solution branches of solitary TWS in panel $4 \mathrm{a}$ are bounded either by the linear thresholds of absolute instability (AI, $\eta=3,3.1$, and 3.4) and full stabilisation (S, $\eta=2$ and 2.8) from panel 2a, or by a nonlinear limit point (LP, $\eta=3.9$ ) that occurs slightly before (about $2 \%$ in terms of $\operatorname{Re}_{g}$ ) the AI bound.

For the strongest confinement, $\eta=2$ (open squares in panel $4 \mathrm{a}$ ), linear and nonlinear effects are aligned and stabilizing. In that case, the gas shapes the wave hump into an elongated flat-top form (panel 4d). In panel 4e, we compare this solution (solid line) with TWS in the limits $\Pi_{\rho}=0$ (red dot-dot-dashed) and $\Pi_{\mu}=0$ (blue dashed), which respectively deactivate the gas pressure and the gas-side viscous stresses in (2.1e) and (2.1f). From this comparison, we can conclude that the gas pressure gradient and not the gaseous viscous stresses are the cause for wave flattening. The flat-top TWS (also shown in figure 1), which we have reproduced with a DNS at slightly greater $\operatorname{Re}_{l}=15.7$ (open circles in panel 4e), is stable in periodic transient computations, and undergoes only weak modulations in a spatially-evolving film (Movie2 . avi).

For the weakest confinement, $\eta=3.9$, solitary TWS are more susceptible to secondary instability modes. We discuss this based on the wave profiles in panel $4 \mathrm{~b}$, which correspond to the TWS marked by open circles in panel 4a. The TWS at $\operatorname{Re}_{g}=-145$ (thick solid profile in 

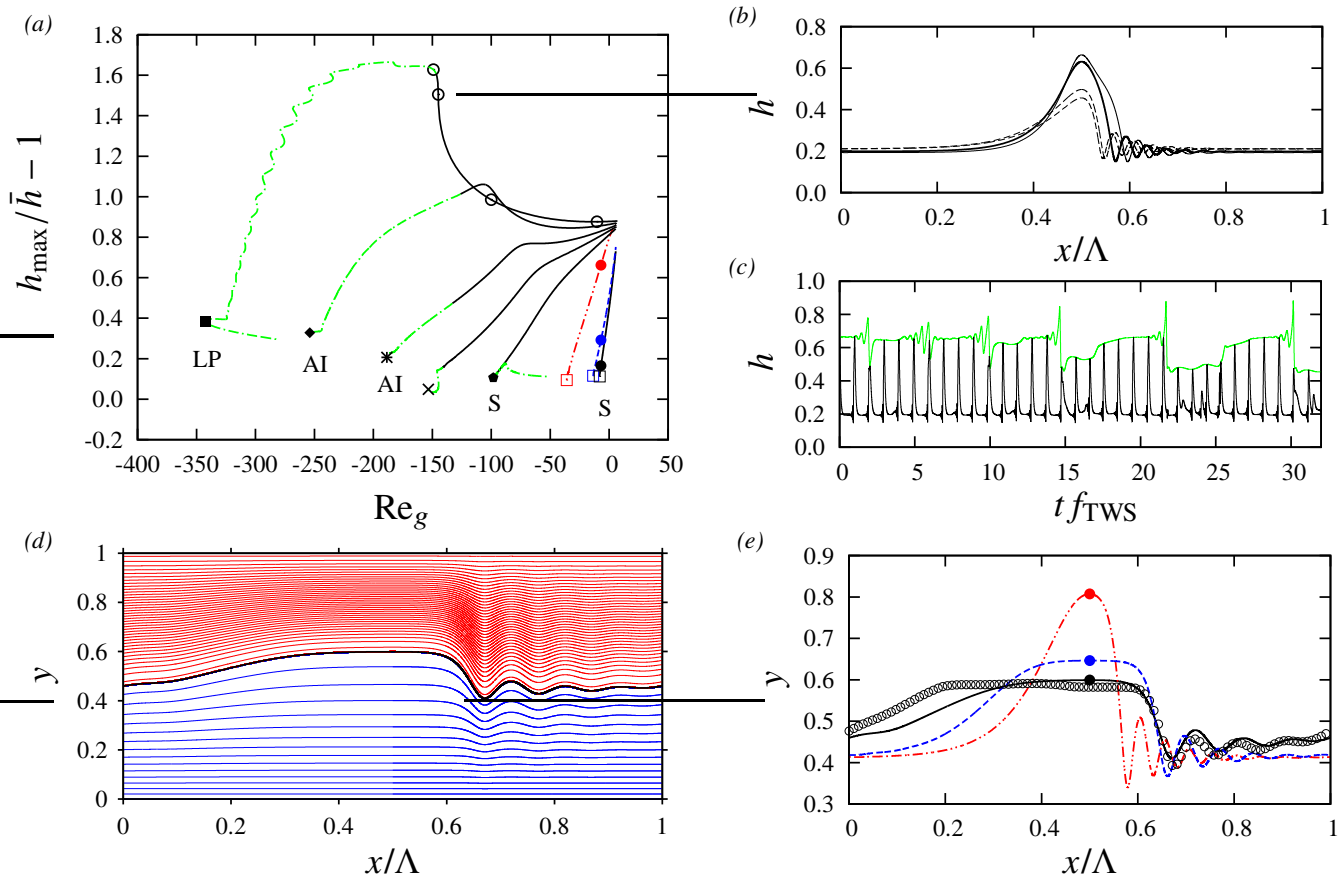

Figure 4: Solitary waves: $\phi=10^{\circ}, \mathrm{Re}_{l}=15, \mathrm{Ka}=509.5, \Lambda=4.5 \tilde{\Lambda}_{\max }$. (a) Amplitude of nonlinear TWS (WRIBL). Right to left: $\eta=2,2\left(\Pi_{\mu}=0\right), 2\left(\Pi_{\rho}=0\right), 2.8,3,3.1,3.4$, and 3.9. Dot-dashed green lines highlight periodically unstable solutions; $(b)$ wave profiles corresponding to open circles $(\eta=3.9)$ in panel $a$. Bottom to top: $\operatorname{Re}_{g}=-10,-100,-145$, and $-149 ;(c)$ transient periodic computation started from thick-solid TWS in panel $b$. Black: local film height, green: wave height; $(d)$ flat-top wave corresponding to black filled circle in panel 4a: $\eta=2, \operatorname{Re}_{g}=-7$. Streamlines in the wave-fixed reference frame; $(e)$ different limits of the $\eta=2$ solution in panel $d$ (filled circles in panel $a$ ). Solid black: full inter-phase coupling; dashed blue: $\Pi_{\mu}=0$ in $(2.1 e)$ and (2.1f); dot-dot-dashed red: $\Pi_{\rho}=0$ in (2.1e); open circles: DNS at $M=\partial_{x} p_{g} / \sin (\phi)=M_{\mathrm{TWS}}=84.8, \operatorname{Re}_{l}=15.7$.

panel 4b, second from left open circle in panel 4a) still lies on the periodically-stable solution branch (solid curve in panel 4a). For this case, secondary instability can only arise through wave interactions. Pradas et al. (2013) showed, for the case of a passive atmosphere, that solitary waves can develop such interactions via the precursory capillary ripples, leading to oscillations around bound states, where neighbouring waves repeatedly approach and recoil from one another. Thereby, the approaching wave always grows, whereas the slowing wave always diminishes in amplitude. In the presence of a counter-current gas flow, we observe a secondary instability mode that involves a different wave interaction. We demonstrate this through an open-domain computation with coherent inlet forcing at the TWS frequency $f=\frac{2 \pi}{\omega}=f_{\text {TWS }}=0.20$. Panels $5 \mathrm{a}$ and $5 \mathrm{~b}$ (see also Movie 3 . avi) show that the instability produces solitary waves of diminishing amplitude that accelerate in the slipstream of their growing leading neighbours. This clearly differs from the behaviour of unconfined falling films, such as the above-mentioned oscillations around bound states (Pradas et al. 2013) or the wellknown coarsening dynamics (Chang et al. 1996b), where larger-amplitude waves catch up with and accumulate the smaller ones travelling in front. The slipstreaming occurs in concert up- and downstream of a leading wave, and thus the latter is increasingly exposed to the counter-current gas flow, leading eventually to its destruction through a wave breaking event, before coalescence can occur.

When increasing the counter-current gas velocity further, TWS become periodically Cambridge University Press 
(a)
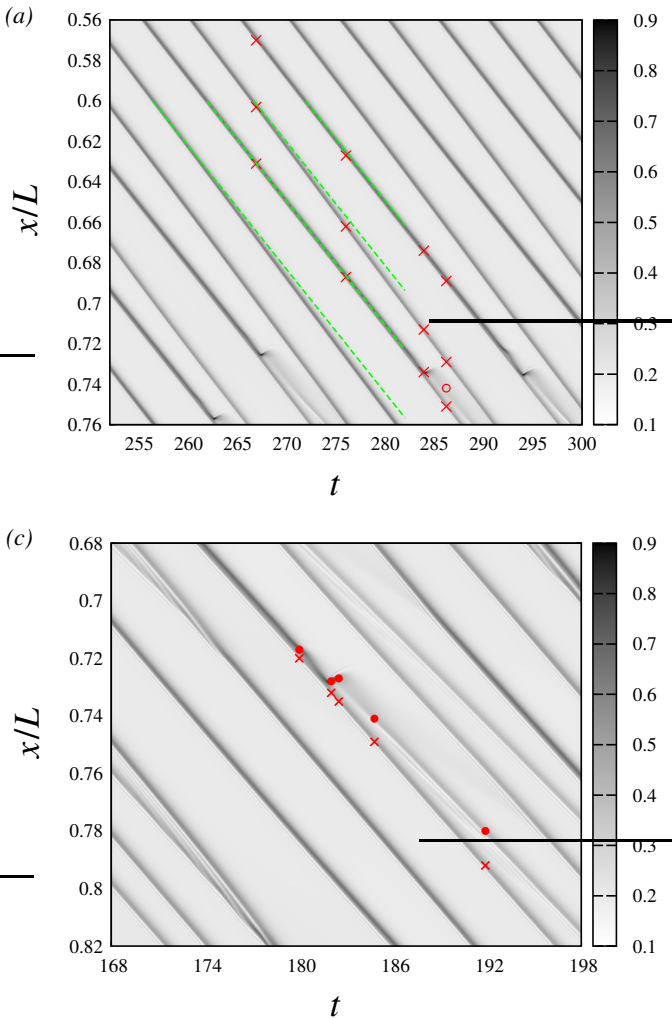

(b)
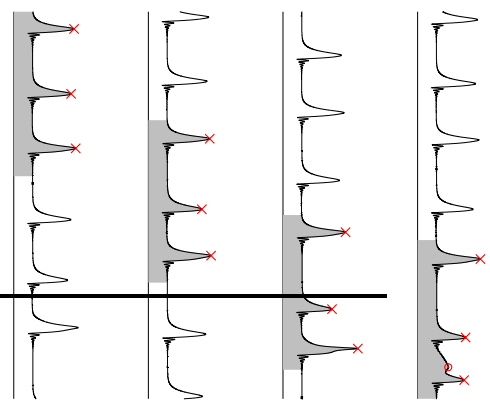

(d)

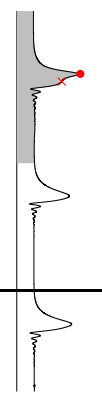

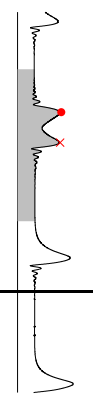
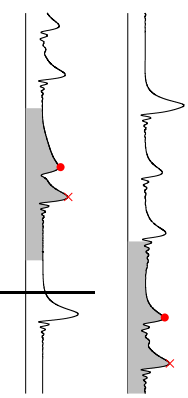

Figure 5: Slip streaming (panels $a, b$ ) and wave splitting (panels $c, d$ ) in solitary wave trains. Spatio-temporal computations with our WRIBL model (2.3) on an open domain of length $L=31.4 \Lambda_{\text {TWS }}$, applying coherent inlet forcing at $f=f_{\text {TWS }}: \phi=10^{\circ}, \eta=3.9, \operatorname{Re}_{l}=15, \mathrm{Ka}=509.5$. Space-time plots of the film height $h$ (panels $a, c$ ), and wave profile snapshots (panels $b, d$ ). Parallel green dashed lines indicate initial TWS celerity. $(a, b) \operatorname{Re}_{g}=-145, f_{\mathrm{TWS}}=0.20 ;(c, d) \operatorname{Re}_{g}=-149, f_{\mathrm{TWS}}=0.19$. Red symbols identify primary/secondary wave maxima.

unstable (dot-dashed branches in panel 4a). For the TWS at $\eta=3.9$ and $\operatorname{Re}_{g}=-149$ (thin solid profile in panel $4 \mathrm{~b}$, leftmost open circle in panel $4 \mathrm{a}$ ), the instability leads to a selfsustained repeated breaking and reconstructing of the wave crest, as shown in panel 4c via a transient computation with periodicity conditions started from the TWS. In a spatially evolving film, which we have mimicked through an open-domain computation with inlet forcing frequency $f=f_{\text {TWS }}=0.19$ (panels $5 \mathrm{c}$ and $5 \mathrm{~d}$, and Movie 4 . avi), the instability leads to ubiquitous wave splitting events that refine the solitary wave train into a train of shorter and smaller daughter waves. This gas-induced refining dynamics can be viewed as the opposite of the coarsening dynamics observed in unconfined films (Chang et al. 1996b). We point out that isolated wave splitting events have been observed in noise-driven wave regimes, both experimentally (Kofman et al. 2017) and numerically (Dietze \& Ruyer-Quil 2013).

\section{Conclusion}

In this work, we have demonstrated that linear stability predictions of strongly-confined falling liquid films can mislead in estimating the effect of a counter-current gas flow on the film's waviness. Both for waves of the most amplified frequency and for low-frequency solitary waves, we have identified situations where the linear and nonlinear responses are 
opposed, i.e. linear waves are damped while nonlinear ones are amplified, or vice-versa. In some cases, linear waves are bounded by absolute instability, whereas nonlinear waves are fully suppressed via a period-halving bifurcation. Nonetheless, at very strong confinement, both the linear and nonlinear responses imply stabilization and travelling-wave solutions (TWS) resist secondary instability. This suggests that the risk of wave-induced flooding can be lowered by strongly confining the flow. At weaker confinement, we have found two new secondary instability modes not observed in unconfined films. The first tends to coarsen the wave train, via smaller waves accelerating in the slipstream of their leading neighbours. The second causes wave splitting events that refine the wave train into a sequence of less dangerous shorter and smaller daughter waves.

Our two-dimensional analysis cannot account for the spanwise destabilization of TWS, which entails the formation of three-dimensional waves in the downstream portion of a spatially-evolving falling liquid film (Chang 1994; Liu et al. 1995; Scheid et al. 2006; Kofman et al. 2014; Dietze et al. 2014; Kharlamov et al. 2015). Nonetheless, we expect our conclusion on the stabilizing effect of strong confinement to extend to that situation. Firstly, the inertia-driven three-dimensional secondary instability mode (Kofman et al. 2014) is known to weaken at the small tilt angles considered here. In experiments, this translates to quasi-two-dimensional wave fronts with only weak spanwise modulations, which are maintained up to large gas velocities (Kofman et al. 2017). Secondly, the spanwise instability mode is dictated by the wall-normal acceleration of liquid within the initially two-dimensional wave hump. Thus, the gas-effect on the amplitude of two- and three-dimensional wave humps is expected to be concurrent. This is supported by the weakly-confined experiments of Kofman et al. (2017), where the counter-current gas flow amplified both instability modes. In our strongly-confined setting, we expect the opposite, i.e. a damping of both modes.

The channel heights considered here $\left(1.2 \mathrm{~mm} \leqslant H^{\star} \leqslant 2.4 \mathrm{~mm}\right)$ lie in between the range of classical (Vlachos et al. 2001) falling-film experiments $\left(H^{\star} \geqslant 5 \mathrm{~mm}\right)$ and micro-channel (Zhang et al. 2009; Hu \& Cubaud 2018) falling-film experiments $\left(H^{\star} \leqslant 1 \mathrm{~mm}\right)$. Also, strongly-confined experiments have generally not considered small tilt angles. Our numerical computations suggest that this uncharted part of the regime map deserves experimental attention. Should experiments confirm our findings, it would mean that surface waves can be maintained in very compact liquid/gas exchangers without the risk of flooding. Current microreactor designs consist of arrays of narrow grooves, where the film surface is pinned laterally (Al-Rawashdeh et al. 2008), and this effectively suppresses surface waves (Pollak et al. 2011), solving the flooding problem, but at the cost of waiving the substantial wave-induced intensification of heat/mass transfer (Yoshimura et al. 1996). Our results suggest relaxing the lateral confinement in such devices to allow for the development of surface waves. Experiments in horizontal wavy liquid-film/gas flows through mini-gaps (Kabov et al. 2007, 2011) have shown that it is possible to produce the strong crosswise confinement levels studied here $\left(H^{\star}=2 \mathrm{~mm}\right)$ at weak spanwise confinement $\left(W^{\star}=40 \mathrm{~mm}\right)$.

\section{Acknowledgements}

We appreciate helpful discussions with J. P. Hulin. This work was supported by the ANR wavyFILM project, grant ANR-15-CE06-0016-01 of the French Agence Nationale de la Recherche.

\section{Declaration of interests}

The authors report no conflict of interest. 


\section{Supplementary data}

The manuscript includes four supplementary movies.

\section{REFERENCES}

Al-Rawashdeh, M., Hessel, V., Löb, P., Mevissen, K. \& Schönfeld, F. 2008 Pseudo 3-d simulation of a falling film microreactor based on realistic channel and film profiles. Chem. Eng. Sci. 63, 5149-5159.

Alekseenko, S. V., Aktershev, S. P., Cherdantsev, A. V., Kharlamov, S. M. \& Markovich, D. M. 2009 Primary instabilities of liquid film flow sheared by turbulent gas stream. Int. J. Multiphase Flow 35, 617-627.

Barmak, I., Gelfgat, A., Vitoshkin, H., Ullman, A. \& Brauner, N. 2016 Stability of stratified two-phase flows in horizontal channels. Phys. Fluids 28, 044101.

Chang, H. C. 1994 Wave evolution on a falling film. Annu. Rev. Fluid Mech. 26, 103-136.

Chang, H. C., Demekhin, E. A. \& Kalaidin, E. 1996a Simulation of noise-driven wave dynamics on a falling film. AIChE J. 42 (6), 1553-1568.

Chang, H. C., Demekhin, E. A., Kalaidin, E. \& Ye, Y. $1996 b$ Coarsening dynamics of falling-film solitary waves. Phys. Rev. E 54 (2), 1467-1477.

DieTZE, G. F. 2019 Effect of wall corrugations on scalar transfer to a wavy falling liquid film. J. Fluid Mech. 859, 1098-1128.

Dietze, G. F., Al-Sibai, F. \& Kneer, R. 2009 Experimental study of flow separation in laminar falling liquid films. J. Fluid Mech. 637, 73-104.

Dietze, G. F., Lavalle, G. \& RuYer-Quil, C. 2020 Falling liquid films in narrow tubes: occlusion scenarios. J. Fluid Mech. 894, A17.

Dietze, G. F., Rohlfs, W., NÄhrich, K., Kneer, R. \& Scheid, B. 2014 Three-dimensional flow structures in laminar falling liquid films. Journal of Fluid Mechanics 743, 75-123.

Dietze, G. F. \& RuYer-Quil, C. 2013 Wavy liquid films in interaction with a confined laminar gas flow. $J$. Fluid Mech. 722, 348-393.

Doedel, E. J. 2008 AUTO07p: Continuation and bifurcation software for ordinary differential equations. Montreal Concordia University .

Drosos, E. I. P., Paras, S. V. \& Karabelas, A. J. 2006 Counter-current gas-liquid flow in a vertical narrow channel - liquid film characteristics and flooding phenomena. Int. J. Multiphase Flow 32, 51-81.

Hu, X. \& Cubaud, T. 2018 Viscous wave breaking and ligament formation in microfluidic systems. Physical Review Letters 121, 044502.

Kabov, O. A., Lyulin, Yu. V., MarchuK, I. V. \& Zaitsev, D.V. 2007 Locally heated shear-driven liquid films in microchannels and minichannels. Int. J. Heat Fluid Flow 28, 103-112.

Kabov, O. A., Zaitsev, D.V., V.V., Cheverda \& Bar-Cohen, A. 2011 Evaporation and flow dynamics of thin, shear-driven liquid films in microgap channels. Exp. Therm Fluid Sci. 35, 825-831.

Kalliadasis, S., Ruyer-Quil, C., Scheid, B. \& Velarde, M. G. 2012 Falling Liquid Films, Applied Mathematical Sciences, vol. 176. Springer Verlag.

Kapitza, P. L. 1948 Wave flow of thin layer of viscous fluid (in Russian). Zhurn. Eksper. Teor. Fiz. 18 (1), $3-28$.

Kharlamov, S. M., Guzanov, V. V., Bobylev, A. V., Alekseenko, S. V. \& Markovich, D. M. 2015 The transition from two-dimensional to three-dimensional waves in falling liquid films: Wave patterns and transverse redistribution of local flow rates. Physics of Fluids 27, 114106.

Kofman, N., Mergui, S. \& Ruyer-Qui, C. 2014 Three-dimensional instabilities of quasi-solitary waves in a falling liquid film. Journal of Fluid Mechanics 757, 854-887.

Kofman, N., Mergui, S. \& Ruyer-Quil, C. 2017 Characteristics of solitary waves on a falling liquid film sheared by a turbulent counter-current gas flow. Int. J. Multiphase Flow 95, 22-34.

Kushnir, R., Barmak, I., Ullmann, A. \& Brauner, N. 2021 Stability of gravity-driven thin-film flow in the presence of an adjacent gas phase. International Journal of Multiphase Flow 135, 103443.

Lavalle, G., Grenier, N., Mergui, S. \& Dietze, G. F. 2020 Solitary waves on superconfined falling liquid films. Physical Review Fluids 5 (3), 032001(R).

Lavalle, G., Li, Y., Mergui, S., Grenier, N. \& Dietze, G. F. 2019 Suppression of the kapitza instability in confined falling liquid films. J. Fluid Mech. 860, 608-639.

Liu, J. \& Gollub, J. P. 1993 Onset of spatially chaotic waves on flowing films. Phys. Rev. Lett. 70 (15), $2289-2292$. 
Liu, J., Schneider, J.B. \& Gollub, J. P. 1995 Three-dimensional instabilities of film flows. Physics of Fluids 7 (1), 55-67.

Pollak, T., Haas, A. \& Aksel, N. 2011 Side wall effects on the instability of thin gravity-driven films from long-wave to short-wave instability. Physics of Fluids 23, 094110.

Popinet, S. 2015 A quadtree-adaptive multigrid solver for the serre-green-naghdi equations. J. Comput. Phys. 302, 336-358.

Pradas, M., Kalliadasis, S., Nguyen, P.-K. \& Bontozoglou, V. 2013 Bound-state formation in interfacial turbulence: direct numerical simulations and theory. Journal of Fluid Mechanics 716, R2.

Ruyer-Quil, C. \& Manneville, P. 1998 Modeling film flows down inclined planes. Eur. Phys. J. B 6 (2), 277-292.

Samanta, A. 2014 Shear-imposed falling film. J. Fluid Mech. 753, 131-149.

Scheid, B., RuYer-Quil, C. \& Manneville, P. 2006 Wave patterns in film flows: modelling and threedimensional waves. J. Fluid Mech. 562, 183-222.

Schmidt, P., NÁraigh, L. Ó., Lucquiaud, M. \& Valluri, P. 2016 Linear and nonlinear instability in vertical counter-current laminar gas-liquid flows. Phys. Fluids 28, 042102.

Sudo, Y. 1996 Mechanism and effects of predominant parameters regarding limitation of falling water in vertical countercurrent two-phase flow. J. Heat Transfer 118 (3), 715-724.

Tilley, B. S., Davis, S. H. \& Bankoff, S. G. 1994 Linear stability theory of two-layer fluid flow in an inclined channel. Phys. Fluids 6 (12), 3906-3922.

TRIFONOv, Y. Y. 2010a Counter-current gas-liquid wavy film flow between the vertical plates analyzed using the Navier-Stokes equations. AIChE J. 56 (8), 1975-1987.

TRIfonov, Y. Y. $2010 b$ Flooding in two-phase counter-current flows: Numerical investigation of the gasliquid wavy interface using the Navier-Stokes equations. Int. J. Multiphase Flow 36, 549-557.

Trifonov, Y. Y. 2017 Instabilities of a gas-liquid flow between two inclined plates analyzed using the Navier-Stokes equations. Int. J. Multiphase Flow 95, 144-154.

TRIFONOv, Y. Y. 2020 Linear and nonlinear instabilities of a co-current gas-liquid flow between two inclined plates analyzed using the Navier-Stokes equations. Int. J. Multiphase Flow 122, 103159.

Tseluiko, D. \& Kalliadasis, S. 2011 Nonlinear waves in counter-current gas-liquid film flow. J. Fluid Mech. 673, 19-59.

Valluri, P., Matar, O. K., Hewitt, G. F. \& Mendes, M. A. 2005 Thin film flow over structured packings at moderate Reynolds numbers. Chem. Eng. Sci. 60, 1965-1975.

Vellingiri, R., Tseluiko, D. \& Kalliadasis, S. 2015 Absolute and convective instabilities in countercurrent gas-liquid film flows. J. Fluid Mech. 763, 166-201.

Vlachos, N. A., Paras, S. V., Mouza, A. A. \& Karabelas, A. J. 2001 Visual observations of flooding in narrow rectangular channels. Int. J. Multiphase Flow 27, 1415-1430.

Yoshimura, P. N., Nosoko, P. \& Nagata, T. 1996 Enhancement of mass transfer into a falling laminar liquid film by two-dimensional surface waves-some experimental observations and modeling. Chem. Eng. Sci. 51 (8), 1231-1240.

Zhang, H., Chen, G., Yue, J. \& Yuan, Q. 2009 Hydrodynamics and mass transfer of gas-liquid flow in a falling film microreactor. AIChE J. 55 (5), 1110-1120. 